\title{
SET MAPPINGS DEFINED ON PAIRS
}

\author{
N. H. WILLIAMS \\ (Received 20 August 1979; revised 15 July 1980) \\ Communicated by J. N. Crossley
}

\begin{abstract}
A set mapping on pairs over the set $S$ is a function $f$ such that for each unordered pair $a$ of elements of $S, f(a)$ is a subset of $S$ disjoint from $a$. A subset $H$ of $S$ is said to be free for $f$ if $x \notin f(\{y, z\})$ for all $x, y, z$ from $H$. In this paper, we investigate conditions imposed on the range of $f$ which ensure that there is a large set free for $f$. For example, we show that if $f$ is defined on a set of size $\kappa^{++}$with always $|f(a)|<\kappa$ then $f$ has a free set of size $\kappa^{+}$if the range of $f$ satisfies the $\kappa$-chain condition, or if any two sets in the range of $f$ have an intersection of size less than $\theta$ for some $\theta$ with $\theta<\kappa$.
\end{abstract}

1980 Mathematics subject classification (Amer. Math. Soc.): 04 A 20, 03 E 05.

\section{Introduction and Notation}

An ordinary set mapping on a set $S$ is a function $f$ mapping $S$ into the powerset $P S$ of $S$ such that $x \notin f(x)$ for each $x$ in $S$. The set map $f$ is said to be of order $\theta$ if $\theta$ is the least cardinal such that $|f(x)|<\theta$ for each $x$ in $S$. A subset $H$ of $S$ is said to be free for $f$ if $x \notin f(y)$ for all $x, y$ in $H$. A classical result (see Erdös (1950), Theorem V) says that every ordinary set map defined on a set of size $\kappa$ and having order less than $\kappa$ has a free set of size $\kappa$. For ordinary set mappings of order $\kappa$ defined on a set of size $\kappa$, extra conditions are needed before a large free set can be guaranteed. Two such conditions considered in the literature are that the range of $f$ should satisfy an intersection condition (see Erdös, Hajnal and Rado (1965), Section 27) or a chain condition (see Erdös, Hajnal and Máté (1973)). The aim of this paper is to obtain results of a similar kind for set mappings defined on pairs.

(C) Copyright Australian Mathematical Society 1981 
First, we introduce our notation. The letters $\kappa, \lambda$ will be used for infinite cardinal numbers, and $\eta, \theta$ for cardinal numbers, finite or infinite. Other lower case Greek letters stand for ordinal numbers. The cardinal numbers are to be identified with the initial ordinals. We use $\kappa^{+}$for the cardinal successor of $\kappa$, and $\kappa^{\prime}$ for the cofinality cardinal of $\kappa$ (so $\kappa^{\prime}$ is the least cardinal $\lambda$ such that $\kappa$ can be expressed as the sum of $\lambda$ cardinals all less then $\kappa$ ). The cardinal $\kappa$ is regular if $\kappa^{\prime}=\kappa$, and otherwise singular. We use $[A]^{\eta}$ or $[A]^{<\eta}$ for $\{B \subseteq A ;|B|=\eta\}$ or $\{B \subseteq A ;|B|<\eta\}$ respectively, and ${ }^{\sigma} A$ (or ${ }^{<} A$ ) for the set of sequences of length $\sigma$ (or less than $\sigma$ ) of elements from $A$. The restriction of the function (or sequence) $f$ to the set $S$ is written $f \uparrow S$. The symbol $\subseteq$ is used for set inclusion, and $\subset$ for proper inclusion.

Definition 1.1. A set mapping on pairs over the set $S$ is a function $f$ : $[S]^{2} \rightarrow P S$ such that $a \cap f(a)=\varnothing$ for each $a$ in $[S]^{2}$. If $\theta$ is the least cardinal such that $|f(a)|<\theta$ for all $a$ in $[S]^{2}$, then $f$ is said to have order $\theta$. A subset $H$ of $S$ is said to be free for $f$ if $x \notin f(\{y, z\})$ for all $x, y, z$ in $H$.

In considering a set mapping $f:[S]^{2} \rightarrow P S$, we shall always suppose that $S$ is a set of ordinals (frequently, $S$ will be a cardinal number); since every set $S$ is in one-to-one correspondence with a set of ordinals, this is no loss of generality. We shall write $f(\alpha, \beta)$ for $f(\{\alpha, \beta\})$, and in the notation $f(\alpha, \beta)$ we shall always assume that $\alpha<\beta$.

Definition 1.2. The family $\underline{A}$ of subsets of the set $S$ is said to satisfy the intersection condition $C(\eta, \theta)$ if $|\cap \underline{B}|<\theta$ for all subfamilies $\underline{B}$ of $\underline{A}$ with $|\underline{B}|=\eta$.

Definition 1.3. The family $A$ of subsets of the set $S$ is said to satisfy the $\tau$-chain condition if there are no sets $D$ contained in $S$ and sequences $\left\langle A_{\alpha}\right.$; $\alpha<\tau\rangle$ of elements of $\underline{A}$ such that $D \cap A_{\alpha} \subset D \cap A_{\beta}$ whenever $\alpha<\beta<\tau$.

We shall assume the Generalized Continuum Hypothesis throughout this paper.

The following results concerned with set maps on pairs appear in Erdös and Hajnal (1958). Every set map $f:\left[\kappa^{++}\right]^{2} \rightarrow P_{\kappa}{ }^{++}$of order at most $\kappa$ has a free set of size $\kappa^{+}$, whereas there is a set map $f:\left[\kappa^{++}\right]^{2} \rightarrow P \kappa^{++}$of order $\kappa^{+}$which has no free set of three elements. In Section 2 we consider set maps $f:\left[\kappa^{++}\right]^{2} \rightarrow$ $\mathrm{P \kappa}^{++}$of order $\kappa^{+}$, and show that $f$ has a free set of size $\kappa^{+}$provided that $\operatorname{ran} f$ satisfies either $C\left(\kappa^{+}, \theta\right)$ for some $\theta$ with $\theta<\kappa$, or the $\kappa^{\prime}$-chain condition (in fact, the conditions we impose are somewhat weaker than these). If instead $\operatorname{ran} f$ satisfies $C\left(\kappa^{+}, \kappa\right)$ or the $\kappa^{+}$-chain condition then $f$ has a free set of size $\kappa^{\prime}$. 
In Section 3, we show how these results can be improved if always the image $f(\alpha, \beta)$ is restricted so that $\alpha \cap f(\alpha, \beta)=\varnothing$. Restrictions of this kind are considered in Hajnal and Máté (1977), Section 4. We show (Theorem 3.1) that every set map $f:\left[\kappa^{+}\right]^{2} \rightarrow \mathrm{P \kappa}^{+}$of order $\kappa$ subject to this restriction has a free set of size $\kappa^{\prime}$. However, if $f$ has order $\kappa^{+}$, no free set of three elements need exist. In Theorem 3.4 we show that if such an $f$ has order $\kappa^{+}$then $f$ has a free set of power $\kappa^{\prime}$ provided that ran $f$ satisfies either the $\kappa$-chain condition or $C(\eta, \kappa)$ for some $\eta$ such that $\lambda^{\eta}<\kappa^{\prime}$ whenever $\lambda<\kappa^{\prime}$.

It will be apparent that our methods extend to give results for set mappings on $n$-tuples for each finite $n$; however, there are sufficiently many open questions concerned just with mappings on pairs that we have not attempted to discuss the case of general $n$.

Most of the results quoted from the literature can be found conveniently either in the survey Hajnal and Máté (1977) or in Williams (1977), Chapter 3.

The main tool that we use is the following particular case of the Ramification Lemma from Erdös, Hajnal and Rado (1965), Lemma 1 (iv) (or see Williams (1977), Theorem 2.2.3 (ii).)

LEMMA 1.4. Let a set $S=S(\varnothing)$ be given, with $|S|=\kappa^{+}$. For all ordinals $\sigma$ with $\sigma<\kappa^{\prime}$, for all sequences $X$ in ${ }^{\sigma} \kappa$ and $Y$ in ${ }^{\sigma+1} \kappa$ let there be given subsets $S(Y)$ and $F(X)$ of $S$, with $|F(X)| \leqslant \kappa$. For each limit ordinal $\sigma$ with $\sigma<\kappa^{\prime}$, for each $X$ in ${ }^{\sigma} \kappa$ define

$$
S(X)=\bigcap\{S(X \uparrow \tau+1) ; \tau<\sigma\} .
$$

For each $X$ in ${ }^{\circ} \kappa$ where $\sigma<\kappa^{\prime}$ put

$$
T(X)=\bigcup\left\{S(Y) ; Y \in{ }^{\sigma+1} \kappa \text { and } Y \uparrow \sigma=X\right\},
$$

and suppose that

$$
S(X)=F(X) \cup T(X) ; \quad F(X) \cap T(X)=\varnothing .
$$

Then there is a sequence $X$ in ${ }^{\alpha^{\prime}} \kappa$ for which $S(X)$ is non-empty.

In the situation of Lemma 1.4, the system of sets $S(X), F(X)$ is called a ramification system on the set $S$, of height $\kappa^{\prime}$ and order $\kappa$.

We conclude this section by noting the following result about ordinary set maps $f: \kappa^{+} \rightarrow \mathrm{P \kappa}^{+}$where $\operatorname{ran} f$ satisfies a chain condition.

THEOREM 1.5. Let $f: \kappa^{+} \rightarrow \mathrm{P \kappa}^{+}$be an ordinary set map of order $\kappa^{+}$such that $\operatorname{ran} f$ satisfies the $\left(\kappa^{\prime}+1\right)$-chain condition. Then $f$ has a free set of size $\kappa^{+}$. 
Proof. By way of contradiction, suppose $f$ has no free set of size $\kappa^{+}$. We shall define a ramification system on $\kappa^{+}$of height $\kappa^{\prime}$ and order $\kappa$ as follows. Take $\sigma$ with $\sigma<\kappa^{\prime}$ and suppose that $S(X)$ has already been defined for all $X$ in ${ }^{\sigma} \kappa$. If $S(X)=\varnothing$, put $F(X)=\varnothing$ and $S(Y)=\varnothing$ for all $Y$ in ${ }^{\sigma+1} \kappa$ where $Y \uparrow \sigma=X$. If $S(X) \neq \varnothing$, choose $G(X)$ to be a maximal free subset of $S(X)$, so $1<|G(X)|<$ к. Put

$$
F(X)=G(X) \cup(S(X) \cap \bigcup\{f(\beta) ; \beta \in G(X)\}),
$$

so $|F(X)|<\kappa$. Note that if $\alpha \in S(X)-F(X)$ then $\alpha \notin \cup\{f(\beta) ; \beta \in G(X)\}$ so by the maximality of $G(X)$, there must be $\beta$ in $G(X)$ for which $\beta \in f(\alpha)$. Write $G(X)=\{\beta(X, \tau) ; \tau<\kappa\}$ and for $Y$ in ${ }^{\sigma+1} \kappa$ with $Y \uparrow \sigma=X$, put

$$
S(Y)=\{\alpha \in S(X)-F(X) ; \beta(X, Y(\sigma)) \in f(\alpha)\} \text {. }
$$

Then $S(X)-F(X)=U\left\{S(Y) ; Y \in{ }^{\sigma+1} \kappa\right.$ and $\left.Y \uparrow \sigma=X\right\}$. This completes the definition of the ramification system.

By Lemma 1.4, we may choose $X$ in ${ }^{\kappa^{\prime}} \kappa$ such that $S(X) \neq \varnothing$. Choose $\beta_{\kappa^{\prime}}$ from $S(X)$. We must have $S(X \uparrow \sigma) \neq \varnothing$ for all $\sigma$, so $\beta(X \uparrow \sigma, X(\sigma))$ will be defined. Put $\beta_{\sigma}=\beta\left(X \uparrow \sigma, X(\sigma)\right.$ ) (for $\sigma$ with $\left.\sigma<\kappa^{\prime}\right)$. If $\sigma<\tau<\kappa^{\prime}$, then $\beta_{\tau} \in S(X \uparrow \tau) \subseteq$ $S(X \uparrow \sigma+1)$ so $\beta_{\sigma}=\beta(X \uparrow \sigma, X(\sigma)) \in f\left(\beta_{\tau}\right)$. Further, $\beta_{\tau} \in S(X \uparrow \sigma+1) \subseteq$ $S(X \uparrow \sigma)$ and $S(X \uparrow \sigma) \cap f\left(\beta_{\sigma}\right) \subseteq F(X\lceil\sigma)$, and $F(X \uparrow \sigma) \cap S(X\lceil\sigma+1)=\varnothing$ so $\beta_{\tau} \notin f\left(\beta_{a}\right)$. Thus if $D=\left\{\beta_{a} ; \sigma<\kappa^{\prime}\right\}$, then

$$
D \cap f\left(\beta_{\tau}\right)=\left\{\beta_{\sigma} ; \sigma<\tau\right\}
$$

so $D \cap f\left(\beta_{\tau}\right) \subset D \cap f\left(\beta_{\mu}\right)$ whenever $\tau<\mu<\kappa^{\prime}+1$, contradicting that $\operatorname{ran} f$ satisfies the $\left(\kappa^{\prime}+1\right)$-chain condition. Hence in fact there must be a free set of size $\kappa^{+}$for $f$.

The chain condition in Theorem 1.5 cannot be weakened if there is still to be a free set of size $\kappa^{+}$. Hajnal (1960), Theorem 1 shows that if $\kappa$ is regular there is an ordinary set map $f: \kappa^{+} \rightarrow P \kappa^{+}$of order $\kappa^{+}$where ran $f$ satisfies the condition $C(2, \kappa)$, which has no free set of power $\kappa^{+}$. Since ran $f$ satisfies $C(2, \kappa)$, it trivially satisfies the $(\kappa+2)$-chain condition. A related result appears in Erdös, Hajnal and Máte (1973), Theorem 3.8, where it is shown in particular that if $f$ : $\boldsymbol{\kappa}^{+} \rightarrow \mathrm{PK}^{+}$is an ordinary set map of order $\kappa^{+}$with ran $f$ satisfying the weaker $\kappa^{+}$-chain condition, then $f$ has a free set of power $\kappa^{\prime}$.

\section{Set mappings on pairs}

In this section, we establish results about set maps $f:\left[\kappa^{++}\right]^{2} \rightarrow P \kappa^{++}$of order $\kappa^{+}$. Theorem 2.1 below is our main tool; it enables results for ordinary set maps 
defined on a set of size $\kappa$ to be "stepped-up" to yield results for set mappings on pairs defined on a set of size $\kappa^{+}$.

THEOREM 2.1. Let $f:\left[\kappa^{+}\right]^{2} \rightarrow P \kappa^{+}$be a set mapping of order at most $\kappa^{+}$. For each $\beta$ with $\kappa \leqslant \beta<\kappa^{+}$and each $B$ from $[\beta]^{\alpha^{\prime}}$ define $f_{\beta, B}: B \rightarrow P B$ by $f_{\beta, B}(\alpha)=$ $B \cap f(\alpha, \beta)$. If every map $f_{\beta, B}$ has a free set of size $\lambda$, then $f$ itself has a free set of size $\lambda$.

Proof. Put $S=\left\{\beta ; \kappa \leqslant \beta<\kappa^{+}\right\}$. We shall define a ramification system on $S$ of height $\kappa^{\prime}$ and order $\kappa$ as follows. Suppose $\sigma<\kappa^{\prime}$ and that $S(X)$ has already been defined for all $X$ in ${ }^{\circ} \kappa$; suppose also ordinals $\beta(Y)$ from $S$ have been defined for all $Y$ in ${ }^{<\sigma_{\kappa}}$. If $S(X)=\varnothing$ put $F(X)=\varnothing, \beta(X)=0$ and for all $Y$ in ${ }^{\sigma+1} \kappa$ with $Y \uparrow \sigma=X$ put $S(Y)=\varnothing$. If $S(X) \neq \varnothing$, define $\beta(X)$ to be the least element of $S(X)$, let $G(X)=\{\beta(X \uparrow \tau) ; \tau \leqslant \sigma\}$ and put

$$
F(X)=\{\beta(X)\} \cup[S(X) \cap \bigcup\{f(\beta(X \uparrow \tau), \beta(X)): \tau<\sigma\}],
$$

so $|F(X)| \leqslant \kappa$. Define a partition $\Delta(X)$ of $S(X)$ by: $y$ and $z$ are in the same class of $\Delta(X) \Leftrightarrow \forall\{\alpha, \beta\} \in[G(X)]^{2}(\alpha \in f(\beta, y) \Leftrightarrow \alpha \in f(\beta, z))$. Then $\Delta(X)$ has at most $2^{|G(X)|}$ classes, so since $|G(X)|<\kappa^{\prime}$ the number of classes is at most $\kappa$. Let $\{S(X, \mu) ; \mu<\kappa\}$ list the classes of $\Delta(X)$. For $Y$ in ${ }^{\sigma+1} \kappa$ with $Y \uparrow \sigma=X$, put

$$
S(Y)=S(X, Y(\sigma))-F(X) .
$$

This completes the definition of the ramification system.

By Lemma 1.4 we may choose $X$ from ${ }^{\kappa^{\prime}} \kappa$ such that $S(X) \neq \varnothing$. In particular, $S(X \uparrow \sigma) \neq \varnothing$ for each $\sigma$ with $\sigma<\kappa^{\prime}$ so $\beta(X \uparrow \sigma)$ is defined; for brevity but $\beta(\sigma)=\beta\left(X\lceil\sigma)\right.$. Also choose $\beta\left(\kappa^{\prime}\right)$ from $S(X)$. When $\mu<\sigma<\tau<\kappa^{\prime}$, since $S(X) \subseteq S(X \uparrow \tau) \subseteq S(X \uparrow \sigma+1)$ both $\beta(\tau), \beta\left(\kappa^{\prime}\right)$ are in $S(X \uparrow \sigma+1)$, which is one of the classes of $\Delta(X \uparrow \sigma)$, so

$$
\begin{aligned}
& \beta(\mu) \in f(\beta(\sigma), \beta(\tau)) \Leftrightarrow \beta(\mu) \in f\left(\beta(\sigma), \beta\left(\kappa^{\prime}\right)\right), \\
& \beta(\sigma) \in f(\beta(\mu), \beta(\tau)) \Leftrightarrow \beta(\sigma) \in f\left(\beta(\mu), \beta\left(\kappa^{\prime}\right)\right) .
\end{aligned}
$$

Further, if $\mu<\sigma \leqslant \kappa^{\prime}$ then $\beta(\mu) \leqslant \beta(\sigma)$ since $\beta(\sigma) \in S(X \uparrow \sigma) \subseteq S(X \uparrow \mu)$ and $\beta(\mu)$ is least in $S(X \uparrow \mu)$; since $\beta(\mu) \in F(X \uparrow \mu)$ then $\beta(\mu) \notin S(X \uparrow \sigma)$ so in fact $\beta(\mu)<\beta(\sigma)$. Put $B=\left\{\beta(\mu) ; \mu<\kappa^{\prime}\right\}$, so $B \in\left[\beta\left(\kappa^{\prime}\right)\right]^{\alpha^{\prime}}$. By hypothesis, we may choose $H$ from $[B]^{\lambda}$ free for $f_{\beta\left(\kappa^{\prime}\right), B}$. In fact $H$ is free for $f$. For take $\beta(\mu), \beta(\sigma)$, $\beta(\tau)$ from $H$, where $\mu<\sigma<\tau<\kappa^{\prime}$. Then $\beta(\tau) \in S(X \uparrow \tau) \in S(X\lceil\sigma+1)$, and $f(\beta(\mu), \beta(\sigma)) \cap S(X\lceil\sigma) \subseteq F(X\lceil\sigma)$ and $F(X\lceil\sigma) \cap S(X \uparrow \sigma+1)=\varnothing$ so $\beta(\tau) \notin f(\beta(\mu), \beta(\sigma))$. Since $H$ is free for $f_{\beta\left(\kappa^{\prime}\right), B}$ we have $\beta(\mu) \notin f\left(\beta(\sigma), \beta\left(\kappa^{\prime}\right)\right)$ and $\beta(\sigma) \notin f\left(\beta(\mu), \beta\left(\kappa^{\prime}\right)\right)$ so by (1) and (2), $\beta(\mu) \notin f(\beta(\sigma), \beta(\tau))$ and $\beta(\sigma) \notin$ $f(\beta(\mu), \beta(\tau))$. Thus $H$ is indeed free for $f$, and the proof is complete. 
The original theorem of Erdös and Hajnal is an easy corollary of Theorem 2.1.

Corollary 2.2 (Erdös and Hajnal (1958), Theorem 3). Every set map $f$ : $\left[\kappa^{++}\right]^{2} \rightarrow P \kappa^{++}$of order $\kappa$ has a free set of power $\kappa^{+}$.

Proof. Apply Theorem 2.1 with $\kappa$ replaced by $\kappa^{+}$, noting $\left(\kappa^{+}\right)^{\prime}=\kappa^{+}$. For any $\beta$ with $\kappa^{+} \leqslant \beta<\kappa^{++}$and any $B$ from $[\beta]^{\kappa^{+}}$, we have that $f_{\beta, B}$ is an ordinary set map of order $\kappa$ defined on a set of size $\kappa^{+}$, and it is well known that every such set map has a free set of size $\kappa^{+}$(Erdös (1950), Theorem V).

As remarked on in Section 1, there is a set map $f:\left[\kappa^{++}\right]^{2} \rightarrow P \kappa^{++}$of order $\kappa^{+}$ which has no free set with three elements, so some further condition on the set maps of order $\kappa^{+}$is needed for the existence of a nontrivial free set. Theorem 2.3 gives several such conditions.

Theorem 2.3. Let $f:\left[\kappa^{++}\right]^{2} \rightarrow P \kappa^{++}$be a set mapping of order $\kappa^{+}$. Suppose whenever $\kappa^{+}<\beta<\kappa^{++}$we have that $\{f(\alpha, \beta) ; \alpha<\beta\}$ satisfies one of

(i) $C\left(\kappa^{+}, \theta\right)$ for some $\theta$ with $\theta<\kappa$,

(ii) the $\left(\kappa^{\prime}+1\right)$-chain condition,

(iii) $C\left(\kappa^{+}, \kappa\right)$,

(iv) the $\kappa^{+}$-chain condition.

If (i) or (ii) hold, $f$ has a free set of size $\kappa^{+}$. If (iii) or (iv) hold, $f$ has a free set of size $\kappa^{\prime}$.

Proof. Take $\beta$ with $\kappa^{+} \leqslant \beta<\kappa^{++}$and take $B$ from $[\beta]^{\kappa^{+}}$. Then $f_{\beta, B}$ is an ordinary set mapping of order $\kappa^{+}$defined on a set of power $\kappa^{+}$. If (i) holds, then $\operatorname{ran} f_{\beta, B}$ satisfies $C\left(\kappa^{+}, \theta\right)$, so by a theorem of Erdös, Hajnal and Rado (1965) (Theorem 36), $f_{\beta, B}$ has a free set of size $\kappa^{+}$. Thus the result follows from Theorem 2.1. If (ii) holds, then $\operatorname{ran} f_{\beta, B}$ satisfies the $\left(\kappa^{\prime}+1\right)$-chain condition and the result follows from Theorems 1.5 and 2.l. If (iii) holds, then ran $f_{B, B}$ satisfies $C\left(\kappa^{+}, \kappa\right)$ and another theorem in Erdös, Hajnal and Rado (1965) (Theorem 46), ensures that $f_{\beta, B}$ has a free set of size $\kappa^{\prime}$, so Theorem 2.1 again applies. If (iv) holds, if follows from Erdös, Hajnal and Máté (1973) (Theorem 3.8(ii)), that $f_{\beta, B}$ has a free set of size $\kappa^{\prime}$, and the result follows.

Some comment is in order as to how close the results in Theorem 2.3 are to being best possible. From Erdös and Hajnal (1958), Theorem 5, we have an example of a set mapping $f:\left[\kappa^{++}\right]^{2} \rightarrow P \kappa^{++}$of order 2 which has no free set of size $\kappa^{++}$. Such a mapping trivially satisfies any of the conditions of Theorem 
2.3, and so shows that a free set of size $\kappa^{+}$is the best that can be expected. If the condition (i) is weakened to $C(2, \kappa)$, I do not know if a free set of size $\kappa^{+}$is still possible. A simple construction from Erdös and Hajnal (1958) provides an example, however, where there is no free set of order type $\kappa^{+}+1$, as follows. For $\beta$ with $\kappa^{+} \leqslant \beta<\kappa^{++}$, by Hajnal (1960), Theorem 1, there is an ordinary set map $f_{\beta}: \beta \rightarrow P \beta$ of order $\kappa^{+}$where $\operatorname{ran} f_{\beta}$ satisfies $C(2, \kappa)$, which has no free set of size $\kappa^{+}$. There is also a set map $g:\left[\kappa^{+}\right]^{2} \rightarrow P \kappa^{+}$of order $\kappa^{+}$which has no free set of three elements, namely $g(\alpha, \beta)=\{\gamma ; \alpha<\gamma<\beta\}$. If $\alpha<\beta<\kappa^{+}$put $f(\alpha, \beta)=g(\alpha, \beta)$; if $\alpha<\beta$ and $\kappa^{+}<\beta<\kappa^{++}$put $f(\alpha, \beta)=f_{\beta}(\alpha)$. Then $f$ has order $\kappa^{+}$and $\{f(\alpha, \beta) ; \alpha<\beta\}$ satisfies $C(2, \kappa)$ whenever $\kappa^{+}<\beta<\kappa^{++}$. Suppose $H$ is a subset of $\kappa^{++}$free for $f$. Then $\left|H \cap \kappa^{+}\right|<2$ since $H \cap \kappa^{+}$is free for $g$. If $\beta \in H$ with $\beta \geqslant \kappa^{+}$then $H \cap \beta$ is free for $f_{\beta}$, so that $|H \cap \beta|<\kappa^{+}$. Hence $H$ can have order type at most $\kappa^{+}$. Moreover, since $\{f(\alpha, \beta) ; \alpha<\beta\}$ satisfies $C(2, \kappa)$, it trivially satisfies the $(\kappa+2)$-chain condition. Hence (for $\kappa$ regular) if (ii) is weakened to the $(\kappa+2)$-chain condition, then a free set of order type $\kappa^{+}+1$ cannot be guaranteed. With regard to (iii), for $\kappa$ regular I do not know if a free set of size $\kappa^{+}$is possible (though the previous example shows that a free set of order type $\kappa^{+}+1$ need not exist). If $\kappa$ is singular, however, using the fact (Erdös, Hajnal and Rado (1965), Theorem 47) that there is an ordinary set map of order $\kappa^{+}$whose range satisfies $C\left(\kappa^{+}, \kappa\right)$ defined on any set of power $\kappa^{+}$which has no free set of size $\left(\kappa^{\prime}\right)^{++}$, a similar construction can be employed to give an example where there is no free set of order type $\left(\kappa^{\prime}\right)^{++}+1$.

\section{Restricted set mappings}

In this section, we consider set maps $f$ on pairs which satisfy the condition $\alpha \cap \beta \cap f(\alpha, \beta)=\varnothing$, that is the image $f(\alpha, \beta)$ always lies above $\alpha$. That this restriction may be relevant is shown by an example from $F$. Galvin (see Hajnal and Máté (1975), p. 358) of a set map $f:\left[\kappa^{+}\right]^{2} \rightarrow P \kappa^{+}$of order $\kappa$ such that $f(\alpha, \beta) \subseteq \alpha$, where $f$ has no infinite free set. The first result shows that under the above restriction, a set map $f:\left[\kappa^{+}\right]^{2} \rightarrow P \kappa^{+}$of order $\kappa$ has a free set of size $\kappa^{\prime}$ (whereas without this restriction, there may be no non-trivial free set).

THEOREM 3.1. Let $f:\left[\kappa^{+}\right]^{2} \rightarrow P \kappa^{+}$be a set map of order $\kappa$ such that always $\alpha \cap \beta \cap f(\alpha, \beta)=\varnothing$. Then $f$ has a free set of power $\kappa^{\prime}$.

Proof. We shall use Theorem 2.1. Take $\beta$ with $\kappa<\beta<\kappa^{+}$and $B$ from $[\beta]^{\alpha}$. Then $f_{\beta, B}$ is an ordinary set map of order $\kappa$ and $\alpha \cap f_{\beta, B}(\alpha)=\varnothing$ for all $\alpha$ in $B$. 
Inductively choose elements $x_{\alpha}$ from $B$ for $\alpha$ with $\alpha<\kappa^{\prime}$ so that

$$
x_{\alpha} \text { is least in } B-\left(\bigcup\left\{f_{\beta, B}\left(x_{\gamma}\right) ; \gamma<\alpha\right\} \cup\left\{x_{\gamma} ; \gamma<\alpha\right\}\right) \text {; }
$$

since always $\left|f_{\beta, B}\left(x_{\gamma}\right)\right|<\kappa$ this is possible. Then $x_{\gamma}<x_{\alpha}$ whenever $\gamma<\alpha<\kappa^{\prime}$, so $x_{\gamma} \notin f_{\beta, B}\left(x_{\alpha}\right)$. Hence $\left\{x_{\alpha} ; \alpha<\kappa^{\prime}\right\}$ is free for $f_{\beta, B}$. The result follows by Theorem 2.1.

In Theorem 3.1 the limitation that $f$ be of order $\kappa$ cannot be lifted to order $\kappa^{+}$. If $f:\left[\kappa^{+}\right]^{2} \rightarrow P_{\kappa^{+}}$is defined by $f(\alpha, \beta)=\{\gamma ; \alpha<\gamma<\beta\}$ then $f$ has order $\kappa^{+}$ with $\alpha \cap \beta \cap f(\alpha, \beta)=\varnothing$, yet $f$ has no free set of 3 elements. However, imposing an intersection condition or a chain condition on the range of $f$ ensures a large free set. There are first a couple of simple lemmas.

Lemma 3.2. Suppose $\lambda^{\eta}<\kappa^{\prime}$. Given families $\underline{A}_{\beta}=\left\{A_{\tau \beta} ; \tau<\lambda\right\}$ where $\beta<\eta$, if $\left|\cap\left\{\cup \underline{A}_{\beta} ; \beta<\eta\right\}\right| \geqslant \kappa$ then there is a function $j: \eta \rightarrow \lambda$ such that $\left|\cap\left\{A_{j(\beta) \beta} ; \beta<\eta\right\}\right| \geqslant \kappa$.

Proof. For each $j: \eta \rightarrow \lambda$ write $A_{j}=\cap\left\{A_{j(\beta) \beta} ; \beta<\eta\right\}$. Then $\mid \cup\left\{A_{j} ; j\right.$ : $\eta \rightarrow \lambda\} \mid>\kappa$ since $\cap\left\{\cup \underline{A}_{\beta} ; \beta<\eta\right\} \subseteq \cup\left\{A_{j} ; j: \eta \rightarrow \lambda\right\}$. Since $\lambda^{\eta}<\kappa^{\prime}$, there must be $j$ with $\left|A_{j}\right| \geqslant \kappa$.

Lemma 3.3 (Máté (1967), Lemma 2). Let $\lambda$ be a regular cardinal and suppose $\underline{A}$ is a family of sets from $[\lambda]^{<\lambda}$ which satisfies the $\lambda$-chain condition. Then there is $\bar{K}$ in $[\lambda]^{<\lambda}$ such that $K \unrhd A$ for all $A$ in $\underline{A}$.

THEOREM 3.4. Let $f:\left[\kappa^{+}\right]^{2} \rightarrow P_{\kappa}{ }^{+}$be a set map of order $\kappa^{+}$such that always $\alpha \cap \beta \cap f(\alpha, \beta)=\varnothing$, and suppose that $\operatorname{ran} f$ satisfies either

(i) $C(\eta, \kappa)$ for some $\eta$ where $\lambda^{\eta}<\kappa^{\prime}$ for all $\lambda<\kappa^{\prime}$, or

(ii) the $\kappa$-chain condition.

Then $F$ has a free set of size $\kappa^{\prime}$.

Proof. We shall define a ramification system on $\kappa^{+}$of height $\kappa^{\prime}$ and order $\kappa$ and also elements $\beta(X)$ of $\kappa^{+}$for $X$ in $<\kappa^{\prime} \kappa^{\prime}$ of length a successor ordinal, as follows. Take $\sigma$ with $\sigma<\kappa^{\prime}$ and suppose that $S(X)$ and $\beta(X \uparrow \tau+1)$ have already been defined for all $X$ in ${ }^{\circ} \kappa$ and $\tau<\sigma$. If $|S(X)|<\kappa^{+}$, put $F(X)=$ $S(X)$ and for all $Y$ in ${ }^{\sigma+1} \kappa$ with $Y \uparrow \sigma=X$ put $S(Y)=\varnothing$ and $\beta(Y)=0$. If $|S(X)|=\kappa^{+}$, for each $\alpha$ in $S(X)$ define

$$
g_{X}(\alpha)=S(X) \cap \bigcup\{f(\beta(X \uparrow \tau+1), \alpha) ; \tau<\sigma\},
$$

so $\left|g_{X}(\alpha)\right|<\kappa$. 
If (i) holds, choose $K(X)$ from $[S(X)]^{\alpha}$, and put $D=\{\alpha \in S(X) ; K(X) \subseteq$ $\left.g_{X}(\alpha)\right\}$. If $|D| \geqslant \eta$, since $|\sigma|^{\eta}<\kappa^{\prime}$, by Lemma 3.2 there would be a function $j$ : $D \rightarrow\{\beta(X \mid \tau+1) ; \tau<\sigma\}$ such that $|\cap\{f(j(\alpha), \alpha) ; \alpha \in D\}| \geqslant \kappa$, contradicting that $\operatorname{ran} f$ satisfies $C(\eta, \kappa)$. Hence $|D|<\eta$. Put

$F(X)=S(X) \cap\left[\bigcup\left\{g_{X}(\alpha) ; \alpha \in K(X)\right\} \cup\{\gamma ; \exists \alpha \in K(X)(\gamma<\alpha)\} \cup D\right]$, so $|F(X)| \leqslant \kappa$.

If (ii) holds, then $\left\{g_{X}(\alpha) ; \alpha \in S(X)\right\}$ satisfies the $\kappa^{+}$-chain condition. For suppose not. Then there are $x_{\xi}, \alpha_{\xi} \in S(X)$ for $\xi$ with $\xi<\kappa^{+}$such that

$$
\xi<\zeta \Leftrightarrow x_{\xi} \in g_{X}\left(a_{\xi}\right) \text {. }
$$

Define a partition of $\left[\kappa^{+}\right]^{2},\left[\kappa^{+}\right]^{2}=\cup\left\{\Delta_{\tau} ; \tau<\sigma\right\}$ by, if $\xi<\zeta$,

$$
\{\xi, \zeta\} \in \Delta_{\tau} \Leftrightarrow x_{\xi} \in f\left(\beta(X \uparrow \tau+1), \alpha_{\zeta}\right) .
$$

Since $|\sigma|<\kappa^{\prime}$ the partition relation $\kappa^{+} \rightarrow(\kappa)_{\sigma}^{2}$ holds (Erdös, Hajnal and Rado (1965), Theorem 2), so there are $H$ in $\left[\kappa^{+}\right]^{\kappa}$ and $\tau$ with $\tau<\sigma$ such that $[H]^{2} \subseteq \Delta_{\tau}$. Thus for $\xi, \zeta$ in $H$,

$$
\xi<\zeta \Leftrightarrow x_{\xi} \in f\left(\beta(X \uparrow \tau+1), \alpha_{\zeta}\right),
$$

contradicting that $\operatorname{ran} f$ satisfies the $\kappa$-chain condition. Thus indeed, $\left\{g_{X}(\alpha) ; \alpha\right.$ $\in S(X)\}$ satisfies the $\kappa^{+}$-chain condition. Then by Lemma 3.3, there is $K(X)$ in $[S(X)]^{\leqslant \kappa}$ such that $K(X) \notin g_{X}(\alpha)$ for all $\alpha$ in $S(X)$. Put

$$
F(X)=S(X) \cap\left[\bigcup\left\{g_{X}(\alpha) ; \alpha \in K(X)\right\} \cup\{\gamma ; \exists \alpha \in K(X)(\gamma<\alpha)\}\right] \text {. }
$$

In either case, note that if $\alpha \in S(X)-F(X)$ then $\alpha>\beta$ for all $\beta$ in $K(X)$, and there is $\beta$ in $K(X)$ such that $\beta \notin g_{X}(\alpha)$. Write $K(X)=\left\{\beta(Y) ; Y \in{ }^{0+1} \kappa\right.$ and $Y \uparrow \sigma=X$ \}, and for $Y$ in ${ }^{\sigma+1} \kappa$ with $Y \uparrow \sigma=X$, put

$$
S(Y)=\left\{\alpha \in S(X)-F(X) ; \beta(Y) \notin g_{X}(\alpha)\right\} .
$$

This determines $\beta(Y)$ and $S(Y)$ for $Y$ in ${ }^{\sigma+1} \kappa$, and completes the inductive definition.

By Lemma 1.4, we may choose $X$ in ${ }^{\prime}{ }^{\prime}$ such that $S(X) \neq \varnothing$. Thus we must have $|S(X \uparrow \sigma)|=\kappa^{+}$whenever $\sigma<\kappa^{\prime}$ so $\beta(X \uparrow \sigma+1)$ is defined. Write $\beta_{\sigma}=$ $\left(X\lceil\sigma+1)\right.$. If $\sigma<\tau<\kappa^{\prime}$ then $\beta_{\sigma} \in K(X \uparrow \sigma)$ so $\left\{\gamma ; \gamma<\beta_{o}\right\} \subseteq F(X \uparrow \sigma)$; since

$$
\beta_{\tau} \in K(X \uparrow \tau) \subseteq S(X \uparrow \tau) \subseteq S(X \uparrow \sigma+1)
$$

and

$$
F(X \uparrow \sigma) \cap S(X \uparrow \sigma+1)=\varnothing
$$

we must have $\beta_{\sigma}<\beta_{\tau}$. Put $H=\left\{\beta_{\sigma} ; \sigma<\kappa^{\prime}\right\}$; I claim that $H$ is free for $f$. For suppose $\sigma<\tau<\mu<\kappa^{\prime}$. Then $\beta_{\sigma}<\beta_{\tau}<\beta_{\mu}$ so $\beta_{\sigma} \notin f\left(\beta_{\tau}, \beta_{\mu}\right)$. Also

$$
f\left(\beta_{o}, \beta_{\tau}\right) \cap S(X \uparrow \tau) \subseteq F(X \uparrow \tau)
$$


and

$$
\beta_{\mu} \in S(X \uparrow \mu) \subseteq S(X \uparrow \tau+1) \subseteq S(X \uparrow \tau)
$$

with $F(X \uparrow \tau) \cap S(X \uparrow \tau+1)=\varnothing$ so $\beta_{\mu} \notin f\left(\beta_{o}, \beta_{\tau}\right)$. Finally,

$$
\beta_{\mu} \in S(X \uparrow \tau+1)-F(X \uparrow \tau+1) \quad \text { and } \quad \beta_{\tau}=\beta(X \uparrow \tau+1)
$$

so

$$
\beta_{\tau} \notin g_{X \mid \tau}\left(\beta_{\mu}\right)=S(X \uparrow \tau) \cap \bigcup\left\{f\left(\beta(X \uparrow \nu+1), \beta_{\mu}\right) ; \nu<\tau\right\} ;
$$

hence $\beta_{\tau} \notin f\left(\beta_{\sigma}, \beta_{\mu}\right)$. Thus $H$ is indeed free for $f$, and the theorem is proved.

\section{References}

P. Erdös (1950), 'Some remarks on set theory', Proc. Amer. Math. Soc. 1, 127-141.

P. Erdös and A. Hajnal (1958), 'On the structure of set-mappings', Acta Math. Acad. Sci. Hungar. 9, 111-131.

P. Erdös, A. Hajnal and A. Máté (1973), 'Chain conditions on set mappings and free sets', Acta Sci. Math. (Szeged) 34, 69-79.

P. Erdös, A. Hajnal and R. Rado (1965), 'Partition relations for cardinal numbers', Acta Math. Acad. Sci. Hungar. 16, 93-196.

A. Hajnal (1960), 'Some results and problems on set theory', Acta Math. Acad. Sci. Hungar. 11, 277-298.

A. Hajnal and A. Máté (1975), 'Set mappings, partitions, and chromatic numbers', Logic Colloquium '73, edited H. E. Rose and J. C. Shepherdson, pp. 347-379 (North Holland, Amsterdam).

A. Máté (1967), 'On the cardinality of strongly almost disjoint systems', Acta Math. Acad. Sci. Hungar. 18, 1-3.

N. H. Williams (1977), Combinatorial set theory (North Holland, Amsterdam, New York, Oxford).

\section{Department of Mathematics}

University of Queensland

St. Lucia, Queensland

Australia 4067 